\title{
System Modeling and Controller Design for Lateral and Longitudinal Motion of F-16
}

\author{
Waqas Ahmed*, Zhongjian Li, Hamid Maqsood, Bilal Anwar \\ School of Automation, Northwestern Polytechnical University, Xi'an, China \\ Email address: \\ Waqas.ahmedk92@gmail.com (W. Ahmed) \\ ${ }^{*}$ Corresponding author
}

To cite this article:

Waqas Ahmed, Zhongjian Li, Hamid Maqsood, Bilal Anwar. System Modeling and Controller Design for Lateral and Longitudinal Motion of F-16. Automation, Control and Intelligent Systems. Vol. 7, No. 1, 2019, pp. 39-45. doi: 10.11648/j.acis.20190701.15

Received: April 15, 2019; Accepted: May 28, 2019; Published: June 15, 2019

\begin{abstract}
Classical control laws are still widely used in aviation industry because of their good structural understanding, simplicity, and better tracking control performance. However in recent decades the application of such controllers are getting substantial interest of researchers. This paper addresses controller design method for longitudinal and lateral motion autopilots of F-16. Aircraft complete mathematical model was obtained using Newton-Euler formulism. The non-linear model was linearized around equilibrium points at certain trim conditions to obtain state space model of the system. Comparative analysis of two linear controllers, Proportional-Integral-Derivative (PID) and Linear-Quadratic-Regulator (LQR) is investigated and control algorithm is proposed. Both the control schemes use feedback control laws and a careful selection of tuning parameters for controllers is carried out to track the desired input reference. Effectiveness of both controllers is illustrated with the help Matlab/Simulink figures and results.
\end{abstract}

Keywords: Aircraft Modeling, Autopilots, PID, LQR

\section{Introduction}

Wright brother's had the honor to make the first flight in aviation history. Following that aviation industry had some dramatic development and the pace with which these changes happened was quite astonishing [1]. The Stability and Control of the airplanes was always a key issue from the very beginning of the aeronautical industry. The introduction of autopilots was one of the great step in aviation development. Initially the autopilots were used with the purpose to replace the human pilot during cruise modes. They were expected to perform more rapidly and with greater precision as compare to the human pilot and to fly aircraft in the same manner as a well-trained pilot. Around 1912 Sperry Gyroscope Company designed and developed an autopilot which was used on a flying boat for a trial. By 1914 the progress reached such a state of development that a public flying demonstration was given [2]. When an aircraft had a deviation form a particular flight path, the autopilots alter the roll, pitch and heading angles of an aircraft. The Automatic Flight Control System (AFSC) concept was changed from mechanically control aircraft to fly by wire control system. In recent decades AFCS is utilized to assist pilot in controlling the aircraft and also help in simultaneously sensors, navigation, guidance and flight instrument display [3]. General aircraft have a six-degree-of-freedom motion, which is further split into translational (horizontal, vertical and transverse) and rotational (pitch, roll and yaw) motions. Aircraft have three control surfaces (Rudder, Elevator and ailerons) which provides help in rotational motion of the aircraft. The lateral axis travel from wingtip-to-wingtip and the pitch motion is angular displacement about this axis while longitudinal axis passes through aircraft from nose-to-tail and motion about this axis is called roll motion. Pitch control can be achieved by providing change to elevator surface. Similarly Roll motion can be controlled with the help of ailerons while for yaw control we need to have a change in rudder surface [4].

In recent decades, enormous techniques including linear and non-linear approaches are investigated to propose control schemes for F-16 [5-7]. It has been stated that LQR controller to be the best for pitch control of aircraft system compared with linear feedback control in [8]. Aircraft Roll Control System Using LQR and PID was done in [9]. 
The motivation behind this work is to design an autopilot based on linear control schemes for F-16 aircraft to control pitch and roll angles. The nonlinear model is linearized around stable equilibrium points and then control laws are investigated and designed to achieve desired response by system states ensuring better control performance, and finite time tracking of roll and pitch angles.

The paper layout is presented as Section 2 discuss the mathematical modelling and linearization of F-16 lateral and longitudinal motion. In section 3 state space representation is presented. Section 4 and 5 present the controllers designing and implementation. Section 6 analyze simulation and results while section 7 ends up the paper with conclusion.

\section{System Modeling and Linearization}

The first step to completely understand and control the aircraft is to obtain the accurate mathematical model. Which can further be used for determining control derivatives and stability. These derivatives alter the flying characteristics and are utilize to control surfaces and design flight control system. There are a lot of ways to obtain the required goal for designing the mathematical model of the aircraft in Matlab/ Simulink, we only described the method which utilized the knowledge of geometry and inertial properties of the system [10].

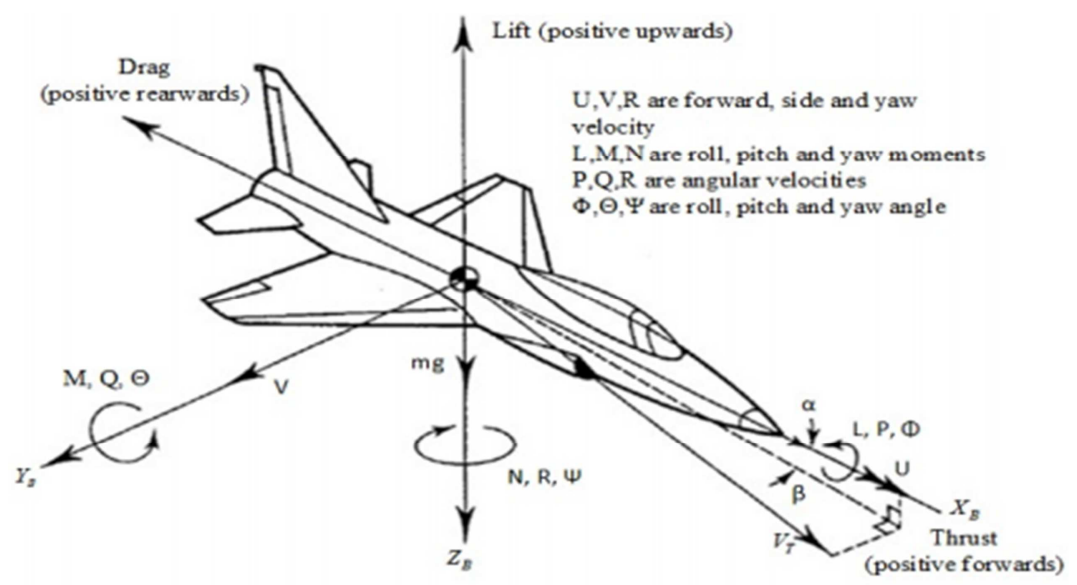

Figure 1. Moments, Euler angles and velocities of Aircraft.

\section{System}

One can easily be familiarize with aircraft model by studying the equations that characterize the motion of the aircraft. These equations can be obtained from the basic laws of kinetic and kinematic. Consider basic newton laws to derive equations for translational and rotational motion. [11]

For the translational dynamics,

$$
\begin{gathered}
\Sigma_{B} F=m \vec{a}_{B} \\
\vec{V}_{B}=\left[\begin{array}{c}
U \\
V \\
W
\end{array}\right] \\
\vec{a}_{B}=\left[\begin{array}{c}
\dot{U} \\
\dot{V} \\
\dot{W}
\end{array}\right]+\left[\begin{array}{c}
P \\
Q \\
R
\end{array}\right] \times\left[\begin{array}{c}
U \\
V \\
W
\end{array}\right]
\end{gathered}
$$

$\left[\begin{array}{lll}U & V & W\end{array}\right]^{T}$ and $\left[\begin{array}{lll}P & Q & R\end{array}\right]^{T}$ Represents translational and rotational velocities of flight.

$$
\vec{F}_{B}=\left[\begin{array}{c}
X \\
Y \\
Z
\end{array}\right]+L_{B V}\left[\begin{array}{c}
0 \\
0 \\
m g
\end{array}\right]
$$

Where $\left[\begin{array}{lll}X & Y & Z\end{array}\right]^{T}$ defines (Lift, Drag, Thrust) acting on Flight and $L_{B V}\left[\begin{array}{lll}0 & 0 & \mathrm{mg}\end{array}\right]^{T}$ represents weight vector Substituting Eq. (3) and (4) in Eq. (1) gives,

$$
\left[\begin{array}{c}
X \\
Y \\
Z
\end{array}\right]+L_{B V}\left[\begin{array}{c}
0 \\
0 \\
m g
\end{array}\right]=m\left(\left[\begin{array}{c}
\dot{U} \\
\dot{V} \\
\dot{W}
\end{array}\right]+\left[\begin{array}{c}
P \\
Q \\
R
\end{array}\right] \times\left[\begin{array}{c}
U \\
V \\
W
\end{array}\right]\right)
$$

After simplifying Eq. (5) given translational dynamics can be achieved for a rigid body.

$$
\left.\begin{array}{l}
X=m(\dot{U}-R V+Q W+g \sin \theta) \\
Y=m(\dot{V}-W P+U R-g \cos \theta \sin \phi) \\
Z=m(\dot{W}-Q U+V P-g \cos \theta \cos \phi)
\end{array}\right\}
$$

For the rotational dynamics of aircraft,

The following moment equations represents the rotational form of Newton's second law.

$$
\begin{aligned}
\Sigma \vec{G}_{B} & =\frac{d \vec{H}_{B}}{d t} \\
\vec{H}_{B} & =I_{B} \cdot \vec{\omega}_{B}
\end{aligned}
$$


$\vec{H}_{B}$ is momentum of the system,

$$
\begin{gathered}
\vec{\omega}_{B}=\left[\begin{array}{c}
P \\
Q \\
R
\end{array}\right] \\
I_{B}=\left[\begin{array}{lll}
I_{X X} & I_{X Y} & I_{X Z} \\
I_{X Y} & I_{Y Y} & I_{Y Z} \\
I_{X Z} & I_{Y Z} & I_{Z Z}
\end{array}\right]
\end{gathered}
$$

$\vec{\omega}_{B}$ And $I_{B}$ are the angular velocity and Moment of inertia of the system respectively,

Substituting Eq. (7), (8) and (9) in Eq. (6) give us the Rotational dynamics of the system.

$$
\left.\begin{array}{l}
L=I_{X} \dot{P}-I_{X Z}(\dot{R}+P Q)+\left(I_{Z}-I_{Y}\right) Q R \\
M=I_{Y}-I_{X Z}\left(P^{2}-R^{2}\right)+\left(I_{X}-I_{Y}\right) P R \\
N=I_{Z} \dot{R}-I_{X Z} \dot{P}-\left(I_{X}-I_{Y}\right)+I_{X Z} R Q
\end{array}\right\}
$$

The above derived translational and rotational equation were used along with disturbance forces and moments, gravitational terms, aerodynamics terms and power terms which are not mentioned here, to get the Longitudinal and lateral directional equations of motion.

\section{State space Representation of Longitudinal and Lateral Equation}

State space is achieved for both longitudinal and lateral motion by trimming the above equations at certain conditions.

$$
\left.\begin{array}{l}
\dot{x}=A x+B u \\
y=C x+D u
\end{array}\right\}
$$

\subsection{Longitudinal Dynamics Model}

The longitudinal dynamics from Eq. (12) are obtained in matrix form as

$$
\begin{gathered}
x=\left[\begin{array}{llll}
\theta & v & \alpha & q
\end{array}\right]^{T} \quad u=\left[\begin{array}{l}
\delta_{e}
\end{array}\right] \quad y=\left[\begin{array}{llll}
\theta & v & \alpha & q
\end{array}\right]^{T} \\
A=\left[\begin{array}{cccc}
0 & 0 & 0 & 1 \\
-32.1 & -0.013 & -2.66 & -1.18 \\
0 & -0.00 & -0.67 & 0.93 \\
0 & 0 & -0.57 & -0.87
\end{array}\right] \quad B=\left[\begin{array}{c}
0 \\
0.0387 \\
-0.0014 \\
-0.1188
\end{array}\right] \\
C=\left[\begin{array}{cccc}
57.2958 & 0 & 0 & 0 \\
0 & 1 & 0 & 0 \\
0 & 0 & 57.2958 & 0 \\
0 & 0 & 0 & 57.2958
\end{array}\right] \quad D=\left[\begin{array}{l}
0 \\
0 \\
0 \\
0
\end{array}\right]
\end{gathered}
$$

\subsection{Lateral Dynamics Model}

The lateral dynamics from Eq. (12) are obtained in matrix form as

$$
\begin{gathered}
x^{T}(t)=\left[\begin{array}{llll}
\phi & \beta & p & r
\end{array}\right] u^{T}(t)=\left[\begin{array}{ll}
\xi & \zeta
\end{array}\right] \\
A=\left[\begin{array}{cccc}
0 & 0 & 1 & 0.078 \\
0.064 & -0.202 & 0.078 & -0.99 \\
0 & -22.92 & -2.25 & 0.54 \\
0 & 6.00 & -0.04 & -0.31
\end{array}\right]
\end{gathered}
$$

$$
B=\left[\begin{array}{cc}
0 & 0 \\
0.0002 & 0.0005 \\
-0.4623 & 0.0569 \\
-0.0244 & -0.0469
\end{array}\right]
$$

$$
C=\left[\begin{array}{cccc}
57.29 & 0 & 0 & 0 \\
0 & 57.29 & 0 & 0 \\
0 & 0 & 57.29 & 0 \\
0 & 0 & 0 & 57.29
\end{array}\right] \quad D=\left[\begin{array}{ll}
0 & 0 \\
0 & 0 \\
0 & 0 \\
0 & 0
\end{array}\right]
$$

\section{Design Process of Longitudinal Controller}

Proportional-Integral-Derivative (PID) and Linear Quadratic Regulator (LQR) are the proposed controllers for pitch control system and are discussed briefly in this section. The general block diagram for pitch attitude control is given below.

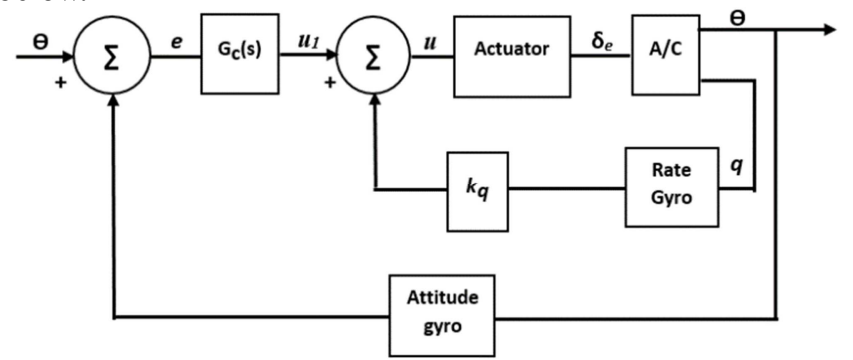

Figure 2. Pitch attitude Autopilot.

\subsection{Proportional-Integral-Derivative (PID)}

PID controller utilizes the basic control scheme of the classical control laws. In basic PID controller measures the error signal value which gives the difference between reference signal and output of the system [12]. The PID controller regulates the pitch control input in order to reduce the error signal. The parameters of PID controller consists of three variables known as the Proportional, the Integral and Derivative $\left(K_{p}, K_{i}\right.$ and $\left.K_{d}\right)$. The output of the PID controller consist of error value, deviation of reference value and oscillation of the system. The working of the system was achieved by properly tuning the value of gains [13]. 


$$
G(s)=K_{p}+K_{i} \frac{1}{s}+K_{d} s
$$

The Proportional gain deal with the loop gain and prevent it from load disturbances. The integral gain component tackle steady state errors and try to minimize it to some extent while the derivative gain tends to achieve the stability of system. The basic requirements of the system output are the stability, peak time, rise time, and percentage overshoot. The requirement of these gains components alter from process to process and is achieved by proper tuning of the PID

parameters $[14,15]$. Here iterative tuning method for PID is done for pitch control of an aircraft.

Table 1. Tuning of PID for Pitch angle Autopilot.

\begin{tabular}{ll}
\hline PID Parameters & Values \\
\hline$K_{p}$ & 6.3 \\
$K_{i}$ & 4.9 \\
$K_{d}$ & 1.4 \\
\hline
\end{tabular}

The Simulink model of PID controller for pitch control is presented below.

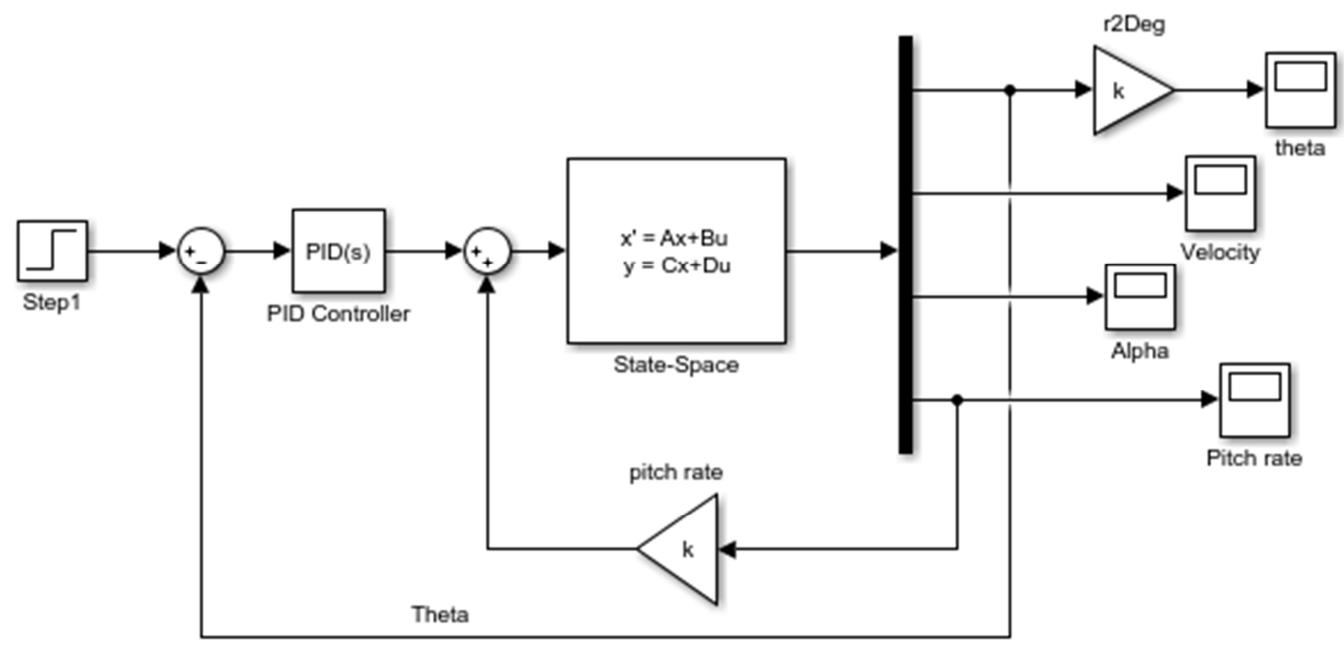

Figure 3. Simulink model of PID Pitch Autopilot.

\subsection{Linear Quadratic Regulator (LQR)}

In last few decades, the classical control laws are replaced by modern control laws, LQR is a controller which utilizes the modern control law and is a powerful controller for almost every linear control system design. During design process of LQR the value of gain ' $K$ ' is selected to make the performance index $\mathrm{J}$ is optimized. This give assurance about the feedback gain ' $\mathrm{K}$ ' optimization for the desired performance index [16].

Performance index $\mathrm{J}$ value was optimized by selecting value of $\mathrm{Q}$ (the weighing function of states) and $\mathrm{R}$ (the weighing function of control variables) [17].

$$
J=\int_{0}^{\infty}\left(x^{T} Q x+u^{T} R u\right) d t
$$

$$
\text { Where } Q=x C^{T} \times C, \mathrm{R}=1
$$

The value of $x$ for $L Q R$ controller is chosen $x=500$ which give the best possible result. Matlab command is used to find the gain matrix K. Value of gain Nbar $=-0.68$ can be easily find with user define matlab code.

$$
K=\operatorname{lqr}(A, B, Q, R)
$$

The obtained gain vector $\mathrm{K}$ is

$$
K=\left[\begin{array}{llll}
-17.2874 & 0.0001 & -5.3117 & -28.0493
\end{array}\right]
$$

Following is Simulink model for LQR controller,

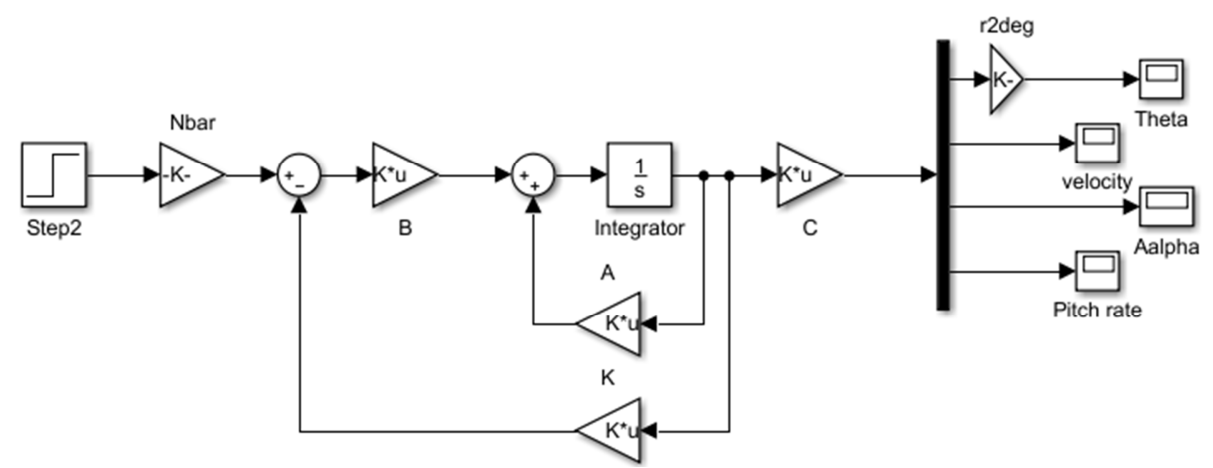

Figure 4. Simulink Model of LQR Pitch Autopilot. 


\section{Design Process of Lateral Controller}

The same PID and LQR controllers which are previously discussed are proposed for lateral motion roll angle control. Figure given below represent a basic roll angle autopilot.

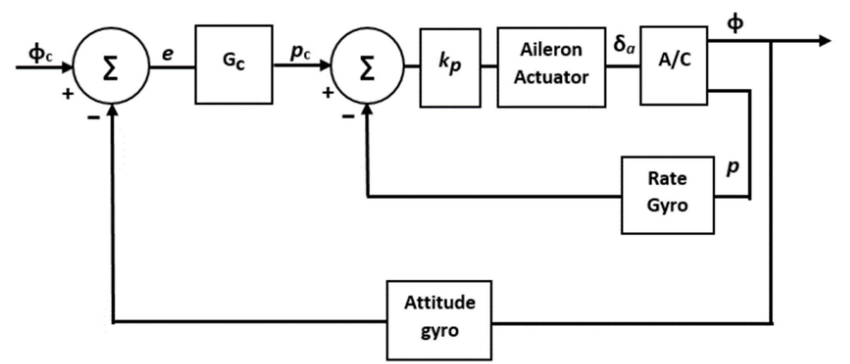

Figure 5. Roll Angle Autopilot.

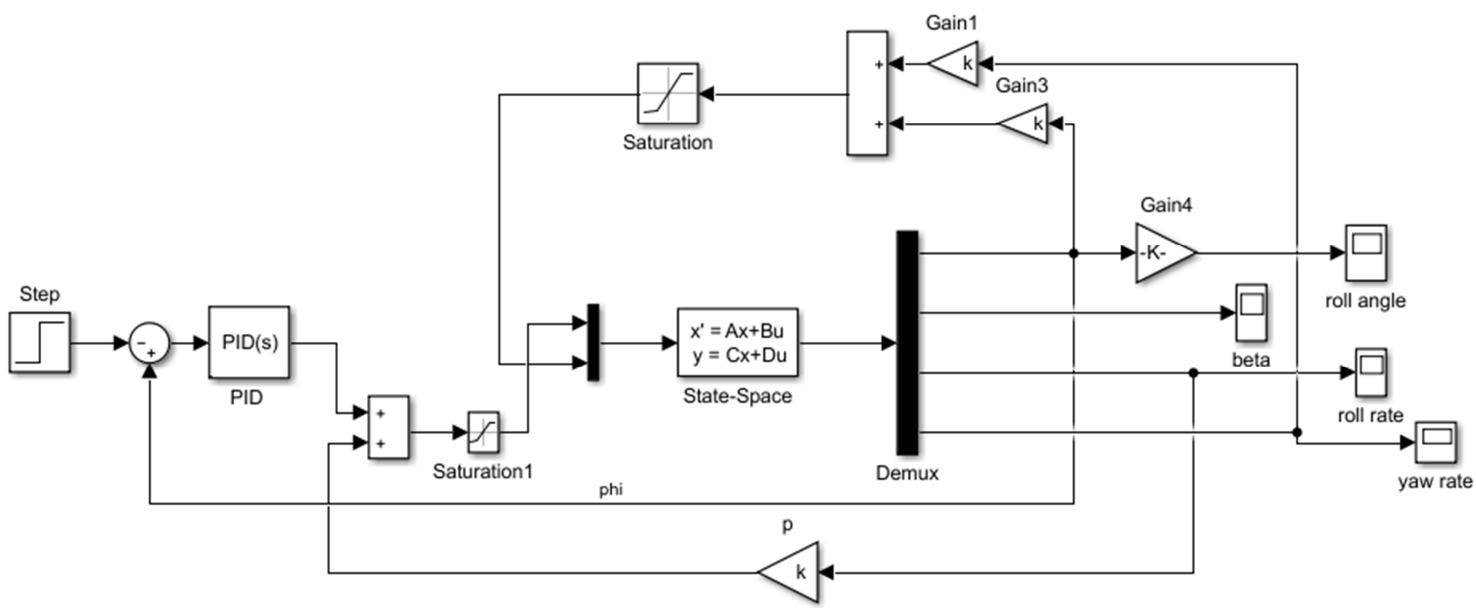

Figure 6. Simulink model of PID Roll angle Autopilot.

\subsection{Linear Quadratic Regulator (LQR)}

LQR is briefly described in section 3, same routine was followed to find the value of gain matrix ' $\mathrm{K}$ '.

Where $Q=x C^{T} \times C, \mathrm{R}=\left[\begin{array}{ll}1 & 1 \\ 0 & 1\end{array}\right]$

\subsection{Proportional-Integral-Derivative (PID)}

PID controller is briefly discussed in last section and here we just implement it to control roll angle. The only difference is that in case of lateral motion we have two inputs controlling roll angle.

Table 2. PID Tuning for Roll angle Autopilot.

\begin{tabular}{ll}
\hline PID Parameters & Values \\
\hline$K_{p}$ & 0.9 \\
$K_{i}$ & 0.6 \\
$K_{d}$ & 0.01 \\
\hline
\end{tabular}

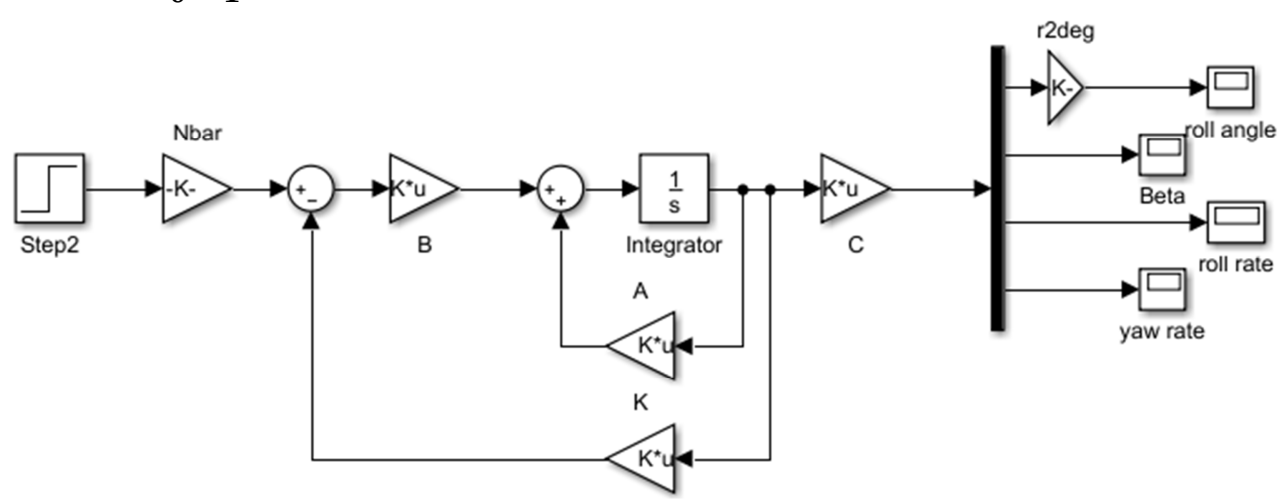

Figure 7. Simulink model of LQR Roll angle Autopilot.

\section{Discussion and Simulation Results}

This study provides a comparative analysis of LQR and PID controllers for pitch and roll control of an aircraft, the
The value of $\mathrm{x}$ for LQR controller is chosen $\mathrm{x}=500$ and

The best possible result. Were obtained. Following command is used to find the gain matrix ' $K$ '. Value of gain Nbar $=-0.2$ can be easily find with user define matlab code. 
and roll response for both control strategies were observed. The system response with proposed controllers indicates the effectiveness of the controllers as the desired references are followed accurately in finite time by the system states.

The performance characteristics are given in Table 3, which prove the effectiveness of the proposed controllers. The LQR controller settling time is higher as compared to PID. The rise time for LQR controller is slightly large as compared to PID. On the other hand peak overshoot for PID is high as compared to LQR controller. Thus the proposed scheme meet the desired references with better control performance and overall satisfactory results in terms of minimum rise time, settling time and percentage overshoot.

Table 3. Comparison of PID and LQR for Longitudinal and Lateral Motion.

\begin{tabular}{llll}
\hline S.No & Settling time & Rise time & Peak Overshoot \\
\hline LQR (longitudinal) & $4.1 \mathrm{~s}$ & $3.5 \mathrm{~s}$ & $0 \%$ \\
PID (longitudinal) & $2.2 \mathrm{~s}$ & $0.3 \mathrm{~s}$ & $25 \%$ \\
LQR (Lateral) & $8.5 \mathrm{~s}$ & $6.1 \mathrm{~s}$ & $0 \%$ \\
PID (Lateral) & $5.1 \mathrm{~s}$ & $0.9 \mathrm{~s}$ & $20 \%$ \\
\hline
\end{tabular}

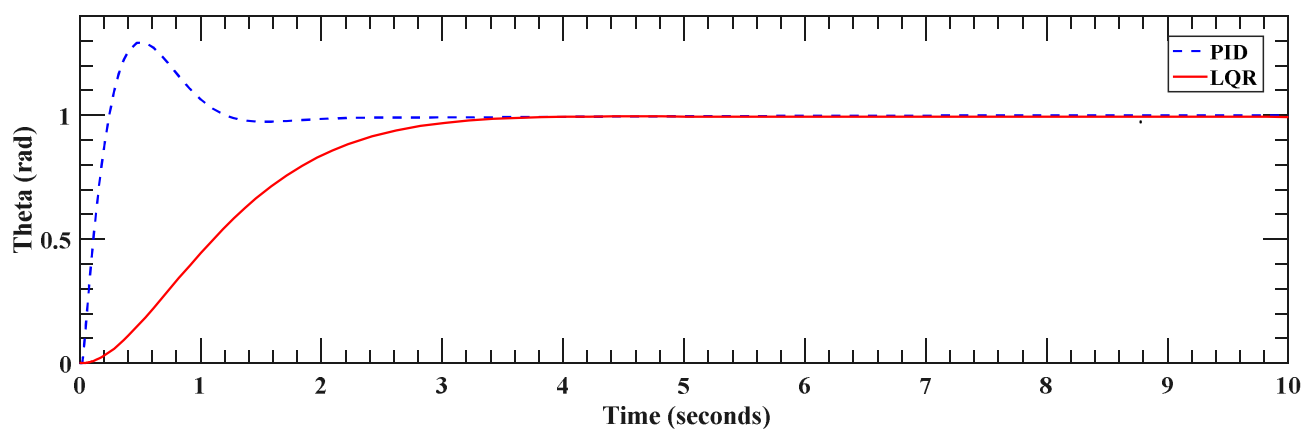

Figure 8. The Performance comparison of $L Q R$ and PID for Pitch angle Autopilot.

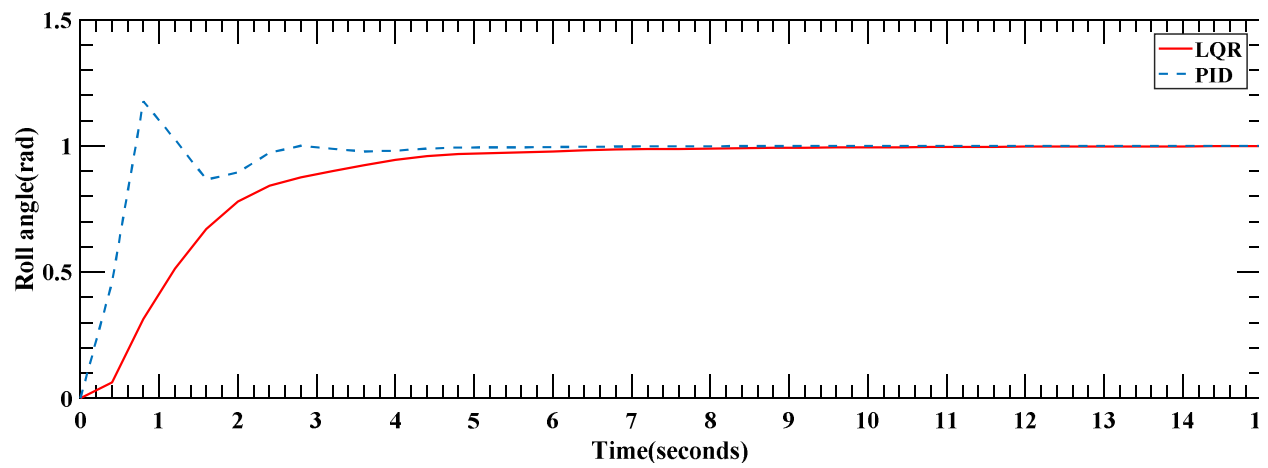

Figure 9. The Performance comparison of $L Q R$ and PID for roll angle Autopilot.

\section{Conclusion}

In this paper, aircraft model for longitudinal and lateral motion was established which was essential in developing the control scheme for autopilots using Matlab/Simulink environment. Successful implementation of two controllers, LQR and PID was carried out here. Simulation results describe that, PID gives a slightly better performance as compare to LQR for settling and rise time. For further research, effort can be done for more robust and advanced controller's development for longitudinal and lateral autopilots.

\section{References}

[1] Stevens, B. L., Lewis, F. L., \& Johnson, E. N. (2015). Aircraft control and simulation: dynamics, control design, and autonomous systems. John Wiley \& Sons.
[2] Ji-Hong Zhu. A Survey of Advanced Flight Control Theory and Application. IMACS Multi conference on Computational Engineering in System Application (CESA). 2006; 1: 655-658.

[3] Z Peng, L Jikai. On new UAV flight control system based on Kalman \& PID. IEEE Transaction, International Conference on Harbin. 2011; 2: 819-823.

[4] McRuer, Duane T., Dunstan Graham, and Irving Ashkenas. Aircraft dynamics and automatic control. Vol. 740. Princeton University Press, 2014.

[5] Ohri, J. (2014, December). GA tuned LQR and PID controller for aircraft pitch control. In 2014 IEEE 6th India International Conference on Power Electronics (IICPE) (pp. 1-6). IEEE.

[6] Usta, M. A., Akyazi, Ö., \& Akpinar, A. S. (2011, June). Aircraft roll control system using LQR and fuzzy logic controller. In 2011 International Symposium on Innovations in Intelligent Systems and Applications (pp. 223-227). IEEE. 
[7] Hajiyev, C., \& Vural, S. Y. (2013). LQR controller with Kalman estimator applied to UAV longitudinal dynamics. Positioning, 4 (1), 36.

[8] Wahid, N., Rahmat, M. F. A., \& Jusoff, K. (2010). Comparative assesment using LQR and fuzzy logic controller for a pitch control system. European Journal of Scientific Research, 42 (2), 184-194.

[9] Rahimi, M. R., Hajighasemi, S., \& Sanaei, D. (2013). Designing and simulation for vertical moving control of UAV system using PID, LQR and Fuzzy Logic. International Journal of Electrical and Computer Engineering, 3 (5), 651.

[10] Noth, A., Bouabdallah, S., \& Siegwart, R. (2006). Dynamic modeling of fixed-wing uavs. Autonomous System Laboratory Report, ETH, Zurich.R.

[11] C. Nelson, 1998, Flight Stability and Automatic Control, McGraw Hill, Second Edition.

[12] Akyazi, O., Usta, M. A., \& Akpinar, A. S. (2012). A self-tuning fuzzy logic controller for aircraft roll control system. International Journal of Control Science and Engineering, 2 (6), 181-188.
[13] X Zhou, Z Wang, $\mathrm{H}$ Wang. Design of Series Leading Correction PID Controller. IEEE Conference. 2009.

[14] G. Sudha and S. N. Deepa "Optimization for PID Control Parameters on Pitch Control of Aircraft Dynamics Based on Tuning Methods" Appl. Math. Inf. Sci. 10, No. 1, 343-350 (2016).

[15] Wahid, N., \& Hassan, N. (2012, February). Self-tuning fuzzy PID controller design for aircraft pitch control. In 2012 Third International Conference on Intelligent Systems Modelling and Simulation (pp. 19-24). IEEE.

[16] Abdulla I. Abdulla, Ibrahim K. Mohammed, Abdulhamed M. Jasim "Roll Control System Design Using Auto Tuning LQR Technique" International Journal of Engineering and Innovative Technology (IJEIT) Volume 7, Issue 1, July 2017.

[17] Labane Chrif, Z. M. Kadda, "Aircraft Control System Using LQG and LQR Controller with Optimal Estimation-Kalman Filter Design," Elsevier, Proce-dia Engineering, 80, (2014): 245. 